ARTíCUlos De INVESTIGACIÓN

\title{
Derecho migratorio: Una respuesta curricular a la sobrepoblación de licenciados en derecho y a las complejidades de su inserción laboral en la región norte de México
}

Lei de imigração: Uma resposta curricular ao elevado número de graduados em direito
e às complexidades de sua inserção no mercado de trabalho na Região Norte do México

Immigration law: A curricular response to the overpopulation of law graduates and the complexities of their labor insertion in the Northern Region of Mexico

\author{
Eduardo Elías Gutiérrez López
}

Universidad Autónoma de Baja California, México

RESUMEN Durante el periodo 2000-2014 en México ha habido un crecimiento exponencial en el número de egresados de las licenciaturas en Derecho - en sus diversas denominaciones- $-y$, por ende, un surgimiento de complejidades en su integración al mercado de trabajo y a condiciones laborales dignas. Este artículo plantea la tesis de que el verdadero problema de este fenómeno no es por sí mismo la proliferación de profesionales del derecho en México, sino la pretensión de todos ellos de emplearse en los mismos nichos de trabajo y desarrollarse en las mismas áreas disciplinares. Esta situación demanda reconfiguraciones a los planes curriculares de la licenciatura en Derecho de las universidades de México y particularmente las de la región norte del país, en las que se focaliza este trabajo y las que, en atención a su situación geográfica, contextual y coyuntural, podrían encontrar en la línea temática del derecho migratorio una respuesta emergente a estas complejidades.

PALABRAS CLAVE Derecho, migración, educación superior, inserción laboral, plan curricular.

RESUMO Durante o período 2000-2014 no México tem havido um crescimento exponencial no número de graduados de licenciaturas em direito - em suas diversas denominações-e, portanto, o surgimento de complexidades na integração de todos eles no mercado de trabalho e em condições de trabalho dignas. Este artigo propõe a tese de que 
o verdadeiro problema deste fenómeno não é por si só a proliferação de profissionais do direito no México, mas a pretensão de todos eles de se empregarem nos mesmos nichos de trabalho e de se desenvolverem nas mesmas áreas disciplinares. Esta situação exige reconfigurações aos planos curriculares da licenciatura em direito das universidades do México e particularmente as da região norte do país, que são aquelas em que se focaliza este trabalho e as que, atendendo à sua situação geográfica, contextual e conjuntural, poderiam encontrar na linha temática direito e migração uma resposta emergente às complexidades mencionadas.

PALAVRAS-CHAVEP Direito, migração, ensino superior, inserção laboral, plano curricular.

ABSTRACT During the period 2000-2014 in Mexico, there has been an exponential growth in the number of graduates of bachelor's degrees in Law, in their various denominations. This growth brought along the emergence of complexities in the integration of all of them into the labor market and decent working conditions. This paper proposes the thesis that the actual problem of this phenomenon is not the proliferation of legal professionals in Mexico, but the intention of most of them to be employed in the same work fields and to perform in the same disciplinary areas. This situation calls for a reconfiguration of the curricula of Mexico's universities. Particularly, those universities in the northern region of the country which are the focus of this work. Given their geographical, contextual, and relevant situation, these Northern universities could find in the thematic line of Law and Migration an emergent response to the complexities mentioned above.

KEYWORDS Law, migration, higher education, job placement, curriculum.

\section{Introducción}

Las escuelas públicas y privadas de educación superior en México se han sobrecargado de estudiantes de las licenciaturas en Derecho - en sus diversas denominaciones- en las últimas décadas y, por lo tanto, el número de abogados o profesionistas en el área legal ha incrementado en forma exponencial. Esta situación representa un reto no solo para las instituciones de educación superior en cuanto a su infraestructura, plantilla de profesores y personal administrativo, sino también al Estado mexicano respecto de la inserción de los egresados de estas licenciaturas al mercado de trabajo y a las condiciones laborales que les esperan en términos salariales, de categoría y áreas de especialización, así como su posible desocupación laboral.

$\mathrm{Al}$ ser demasiados quienes egresan de estos programas educativos por semestre o en ocasiones por cuatrimestre - según sea el plan de estudios de la institución académica一, se complica su integración al mercado laboral y se precarizan las condiciones 
de trabajo que les ofrecen los empleadores. Por lo general, la inserción de un egresado de la licenciatura en Derecho se reduce a tres grandes campos: i) sector público, en tanto trabaje para alguna institución del Estado o universidad pública; ii) sector privado, al adherirse a algún bufete jurídico o empresa; y iii) sector social, que abarca a las organizaciones de la sociedad civil.

Sin embargo, las instituciones del sector público se han quedado cortas en relación con la demanda de trabajo que requieren los egresados de Derecho en México, pues, entre quienes ya son profesionistas de tiempo y los que cada cuatro o seis meses egresan de las instituciones de educación superior al mercado laboral, se vuelve un número imposible de insertar por completo al sector público. A su vez, el sector privado, aunque requiere de abogados recién egresados, no está en condiciones de ofrecer oportunidades de trabajo óptimas (ingresos por encima de los $\$ 12.000$ pesos mexicanos mensuales, seguridad social, prestaciones laborales, etcétera). Por último, las organizaciones de la sociedad civil, como parte del sector social, al ser asociaciones sin finalidades de lucro, tampoco reúnen los elementos para ofrecer condiciones laborales sólidas y responder al cúmulo de egresados de Derecho en búsqueda de empleo en México.

Debemos recordar en todo momento que una de las misiones nodales de la Organización Internacional del Trabajo, como agencia de Naciones Unidas, es la de promover oportunidades para la obtención de trabajos decentes (Ghai, 2003). El trabajo decente debe cumplir con cuatro requisitos primordiales: el empleo, la protección social, los derechos de los trabajadores y el diálogo social (Ghai, 2003); por consiguiente, estos elementos tienen que estar presentes en las relaciones laborales en las que participen los egresados de las licenciaturas en Derecho en México.

A pesar de este panorama adverso en cuanto a la situación de los licenciados -al menos desde el ámbito de integración laboral-, este trabajo parte de dos preguntas de investigación: ¿cuáles son las razones por las que se ha complejizado la integración laboral de los egresados de las licenciaturas en Derecho en el norte de México? y ¿cómo podría contrarrestarse esta situación? Por lo tanto, su objetivo radica en plantear la tesis de que el problema no se encuentra en la sobrepoblación per se, sino en el hecho de que la gran mayoría de egresados de esta disciplina profesional pretenden desarrollarse en las mismas áreas disciplinares, muchas veces porque así fueron instruidos. Por ello, el segundo objetivo de este trabajo estriba en advertir la necesidad de reconfigurar los planes de estudio de las universidades públicas que ofrecen la licenciatura en Derecho en México y abrir el espacio para nuevas áreas disciplinares, entre ellas el área del derecho migratorio en sus diferentes vertientes. Esto con el propósito, en primer término, de no sobrepoblar aquellas áreas que suelen ser más trabajadas, pero también para dar lugar a temas coyunturales en los que se requiere de expertos y hay pocos pujando por obtener dichos espacios, mientras el resto sigue buscando en las ramas tradicionales del derecho en México: civil, penal y laboral. 
En la metodología de este trabajo, además de revisar las cifras respecto de las instituciones de educación superior que ofertan la licenciatura en Derecho en México y los números en cuanto a cédulas profesionales se refiere para ejercer esta carrera profesional, se han analizado algunas de las características principales de los planes de estudio de estas ofertas académicas, en específico los de las universidades públicas de la frontera norte de México. Asimismo, se hicieron dos entrevistas semiestructuradas a profesionistas del derecho en el norte de México que se desempeñan en el derecho migratorio, con el propósito de subrayar el mercado de trabajo en el que podrían integrarse los abogados que decidieran especializarse en esta rama profesional dentro del contexto de esta zona geográfica. La razón para la selección de estas instituciones académicas públicas versa en que, de este modo, es metodológicamente más viable el análisis, al ser demasiadas los programas de la licenciatura en Derecho que se ofrecen en el país, aunado que al tratarse de proponer la pertinencia de la línea temática de derecho migratorio - en sus diversas vertientes- a estos programas, esta misión debe estar concentrada en el norte del país por su posición fronteriza y en las instituciones públicas, pues éstas, bajo el nuevo modelo universitario, tienen como finalidad institucional la responsabilidad social, y, por lo general, son un referente para las escuelas privadas, por lo que los cambios vislumbrados en ellas suelen incidir en las universidades del sector privado.

\section{Las cifras de los licenciados en Derecho en México: Un desafío que demanda acciones inmediatas}

De acuerdo con los datos del Centro de Estudios sobre la Enseñanza y el Aprendizaje de Derecho para el ciclo académico 2018-2019 (CEEAD, 2018), en México estaban activas 1.911 instituciones de educación superior que ofrecían la licenciatura en Derecho en cualquiera de sus denominaciones, de las cuales 1.734 son privadas y 177 públicas. No obstante, existen otras 340 instituciones que, sin estar activas, tienen autorización para impartir la licenciatura en Derecho: 149 están cerradas temporalmente pero con posibilidades de reactivarse, y 191 cerradas en forma definitiva.

Lo anterior muestra el gran número de instituciones que ofertan la licenciatura, pero también lo sencillo que es para las escuelas privadas obtener la autorización para ofrecerla, en gran medida por los pocos requisitos que se solicitan, a diferencia de otras disciplinas. Por ejemplo, las ciencias de la salud, además de cumplir con los lineamientos propuestos en el instructivo general del Acuerdo 279, por el que se establecen los trámites y procedimientos relacionados con el reconocimiento de validez oficial de estudios del tipo superior, deben pedir la opinión de la Comisión Interinstitucional para la Formación de los Recursos Humanos para la Salud, organismo colegiado interinstitucional, de consulta, asesoría y apoyo técnico de la Secretaría de 
Educación Pública y de la Secretaría de Salud para identificar las áreas de coordinación entre las instituciones educativas y las de salud.

Por su parte, en el caso de las ingenierías, deben cumplir con un requerimiento especial que consiste en adminicular su programa de investigación a seguir y la relación de equipamiento para su desarrollo, así como del personal a su cargo. Estas distinciones obedecen a la clasificación que efectúa el Estado mexicano en dicho Acuerdo 279, en el que entrega a la licenciatura en Derecho la categoría de «programa práctico» y, por ende, el no requerimiento de muchos cursos básicos en ciencias o humanidades, ni tampoco cursos con gran tiempo de atención por alumno, lo que representa más facilidad para obtener el reconocimiento de validez.

A estas altas cifras de las instituciones académicas se agrega la cuestión de su calidad, ya que solo el 6\% de las que cuentan con autorización de la Secretaría de Educación Pública para ofertar la licenciatura tiene acreditado su programa académico ante algún organismo evaluador (CEEAD, 2018), como el Consejo Nacional para la Acreditación de la Educación Superior en Derecho (CONFEDE), el Consejo para la Acreditación de la Enseñanza del Derecho (CONAED) y los Comités Interinstitucionales para la Evaluación de la Educación Superior (CIES), entre otros. Esto significa que no solo habrá muchos egresados de estas disciplinas, sino que la mayor parte de ellos se habrá instruido en alguna escuela o institución sin un programa de calidad o debidamente acreditado. De acuerdo con Medina y Valdez (2011), la importancia de los procesos de acreditación de los programas de estudio se encuentra en que a través de estos mecanismos se logra, en un primer momento, orientar las actividades de formación de los egresados y, en un segundo momento, mejorar constantemente la calidad de las universidades.

Los problemas de calidad y acreditación por algún organismo evaluador no son una característica propia de los programas de licenciatura en Derecho de las universidades de México, pues incluso si se extiende el análisis a las ciencias sociales en general, encontraremos la misma situación. Aunque son muchas las universidades públicas que ofertan programas educativos en ciencias sociales, muy pocas tienen programas de licenciatura acreditados, así como posgrados de calidad (Contreras y Hualde, 2015).

A pesar de lo anterior, y seguramente por la gran sobrepoblación de egresados, derecho es la disciplina de las ciencias sociales ${ }^{1}$ que más profesionistas ocupados laboralmente tiene, con 791.386, y de todas las disciplinas es la segunda, solo por debajo de administración y gestión de empresas, que cuenta con 953.007.2

1. Se reconoce la discusión existente sobre si el derecho forma parte o no de las ciencias sociales, pero al final en la Encuesta Nacional de Ocupación y Empleo es colocada dentro de estas áreas disciplinarias.

2. Secretaría del Trabajo y Previsión Social e Instituto Nacional de Estadística y Geografía, «Encuesta Nacional de Ocupación y Empleo», cifras actualizadas al segundo trimestre de 2019. 
Ahora bien, de todas estas instituciones de educación superior que ofrecen la licenciatura en Derecho, se debe analizar cuántos egresan y, más relevante aún, cuántos están obteniendo su cédula profesional para ejercer. Entre los periodos 2000-2004 al 2010-2014 hubo un crecimiento de casi el doble de cédulas profesionales registradas y expedidas por la Dirección General de Profesiones en México para ejercer la licenciatura en Derecho, es decir, de 98.141 a 179.790 (CEEAD, 2018).

Con todas las limitaciones y sesgos metodológicos que esta información pueda tener ante la posibilidad de que muchas personas con cédulas profesionales ya hayan fallecido o no se encuentren ejerciendo su profesión, los números son considerablemente altos de cualquier modo. De esta manera, la inserción al mercado de trabajo de los egresados de la licenciatura en Derecho constituye un desafío para México, pues más allá de que 791.386 se encuentren ocupados laboralmente — según la Encuesta Nacional de Ocupación y Empleo-, el promedio del ingreso mensual de estos trabajos es de \$12.101 pesos mexicanos, es decir, muy reducido para el costo de vida que tiene el país.

Como se puede apreciar, las cifras en la materia son contundentes y no proyectan un panorama alentador para quienes egresan de estas carreras académicas. Por un lado, son demasiadas las instituciones de educación superior que están en condiciones legales de ofertar estas licenciaturas por la facilidad de los mismos lineamientos educativos; por el otro, una enorme mayoría de programas académicos no se encuentran acreditados en cuanto a su calidad por algún organismo evaluador.

Aunque las estadísticas demuestran que derecho es la segunda disciplina profesional con más personas ocupadas laboralmente en México, esto puede ser por la gran cantidad de profesionistas que egresan y obtienen su cédula cada semestre o cuatrimestre, además de que el ingreso mensual no es acorde al costo de vida del país o al número de cédulas profesionales expedidas de licenciatura en Derecho a partir del periodo 2000-2014, las que han crecido en forma exponencial. Por todo esto, las cifras se vuelven un reto para el Estado mexicano, que debe encontrar una manera de asegurar las condiciones de trabajo mínimas y dignas para que los egresados puedan ocuparse laboralmente, emprender sus propios negocios o integrarse a unidades de producción que ya se encuentren activas.

\section{Conexiones entre los planes curriculares de las profesiones jurídicas y la integración laboral: Construyendo perfiles}

A partir del ingreso del modelo por competencias a las instituciones de educación superior, se consolidó un cambio de perspectiva en la forma de enseñanza y se construyó una nueva dinámica al interior de las aulas. El modelo por competencias es un enfoque que «busca que los estudiantes de educación básica desarrollen habilidades para poner en práctica el conocimiento en la resolución de problemas de la vida cotidiana» (Barraza, 2016: 40). 
En el caso de las disciplinas jurídicas, de acuerdo con González y Muñoz (2010), los alumnos pasaron a ser los protagonistas del proceso de enseñanza-aprendizaje y la instrucción transitó de concentrarse en la amplitud de conocimientos jurídicos a la capacitación en habilidades y destrezas que pudieran ser requeridas en el ejercicio profesional. En otras palabras, se tejió una conexión más sólida entre los planes curriculares, la forma de operarlos en el aula y la integración laboral de los estudiantes en el futuro.

Es por ello que cada vez se ven más planes de estudio de las licenciaturas en Derecho que tienen como común denominador la constante preocupación de incluir asignaturas o cursos que contemplen el modo de enseñar estas destrezas y habilidades pragmáticas. Por ejemplo, seminarios prácticos, asignaturas enfatizadas en los apartados procesales de las diferentes disciplinas del derecho y prácticas profesionales en las que los estudiantes deben incluso salir de las universidades y desempeñar por un periodo corto actividades vinculadas con su profesión en algún bufete jurídico, empresa u organización de la sociedad civil.

Sin embargo, para efectos de pensar en un proceso de integración laboral de los estudiantes, es menester contemplar los requerimientos del mercado de trabajo, los que inexorablemente varían en función de factores geográficos y económicos de cada región. Lo anterior no debe ser ajeno a las preocupaciones de la educación jurídica, pues como lo señalan González y otros (2011), existen tres ejes nodales dentro del estado del arte de la educación jurídica: i) la universidad como institución formadora, la carrera y la formación de los abogados; ii) la universidad y el «afuera»; y iii) la institución y el campo universitario.

El segundo eje comprende, además de otros aspectos, lo concerniente a la realidad social y al mercado de trabajo (González y otros, 2011), dos componentes cardinales del fenómeno migratorio y su conectividad con la frontera norte de México. En el estado del arte de la educación jurídica ha quedado de manifiesto que gran parte de los desafíos que tienen trazados las instituciones educativas superiores en materia de formación jurídica estriban en la necesidad de adaptar sus prácticas de enseñanza a lo que sucede y se demanda en el exterior.

En otras palabras, se debe dar coherencia a la relación existente entre la universidad y el «afuera» (González y otros, 2011), de lo contrario, no solo se pierde la oportunidad de mejorar el análisis, la gestión y regulación de esa realidad social, sino que además se diluyen espacios de trabajo que podrían ser ocupados por los egresados de las licenciaturas en Derecho. De acuerdo con Camarena y Velarde (2009), existen dos vertientes a partir de las cuales pueden ser comprendidos los propósitos de las universidades: por un lado, una vertiente pragmática que da cuenta de la construcción de una plataforma de profesionistas que puedan responder a los requerimientos del mercado de trabajo; y, por otro lado, una vertiente ética que corresponde al posicionamiento universitario respecto de los diferentes fenómenos políticos, económicos y sociales que convergen en la realidad. 
De tal suerte, preocuparse por el «afuera» no solo implica atender las necesidades de los mercados laborales, sino también el compromiso de las instituciones de educación superior de coadyuvar con los Gobiernos en turno y con la misma sociedad en la resolución de problemáticas sociales y en la instauración de un mejor entendimiento de muchos de los fenómenos que se presentan en la realidad de las diferentes comunidades, ciudades y países. La responsabilidad social es un atributo que las instituciones educativas tomaron desde 1990 del sector empresarial (Collazo, 2014) y que consiste en el compromiso de las universidades por ayudar a las comunidades a la definición de sus prioridades en aras de lograr un desarrollo sostenible (Neave, 1998).

A esto habría que agregar que, en términos de algunas propuestas sobre teoría curricular, como la de Hilda Taba (citado en Díaz, 1993), se define que los programas escolares deben tener como referentes la cultura y la sociedad. De allí que se torne imprescindible elaborar un diagnóstico de necesidades sociales que sustente el contenido del diseño curricular, uno que además permita que sus universitarios incidan en las políticas públicas y en los grandes retos socioeconómicos y ambientales (Vallaeys, 2014).

Es por todo lo anterior por lo que resulta menester aportar con los diseños curriculares de las licenciaturas en Derecho a esos dos componentes de la educación jurídica. En primer término, a lo concerniente a fortalecer la conexión entre los profesionistas que se forman en las universidades y los principales requerimientos del mercado laboral dentro del área jurídica. En segundo término, lo relativo a la responsabilidad social que deben asumir las universidades como parte de sus nuevos atributos educativos en el marco del desarrollo y la sostenibilidad, para abonar en la medida de sus posibilidades y campos de acción, a las necesidades de índole social y a los diferentes fenómenos que demandan participación y análisis para su mejor gestión y entendimiento.

\section{Los planes de estudios de la licenciatura en Derecho de las universidades mexicanas: El tópico pendiente de la educación superior}

Si tomamos en consideración que México se encuentra compuesto por 31 entidades federativas y la Ciudad de México, cada una de ellas con diferentes universidades públicas y privadas, a efecto de simplificar el análisis y la metodología empleada y sobre todo para justificar la importancia del estudio del fenómeno migratorio desde la óptica del derecho, se circunscribió el estudio a los planes de estudio de las instituciones de educación superior públicas dentro de las entidades federativas de la frontera norte de México: Baja California, Sonora, Chihuahua, Coahuila, Nuevo León y Tamaulipas. Se revisaron los planes de estudio de la licenciatura en Derecho de las siguientes universidades: Universidad Autónoma de Baja California (UABC), Universidad de Sonora (UNISON), Universidad Autónoma de Chihuahua (UACH), 
Universidad Autónoma de Coahuila (UAdeC), Universidad Autónoma de Nuevo León (UANL) y Universidad Autónoma de Tamaulipas (UAT).

Como se ha explicado en la parte introductoria de este trabajo, se optó por universidades del sector público porque se estima que éstas son las que principalmente poseen un compromiso de responsabilidad social con sus comunidades emanado de los nuevos modelos educativos; parte de este compromiso radica en formar estudiantes que sean capaces de contribuir a la resolución de problemáticas sociales y a la consolidación de una cultura de paz, de legalidad y de respeto por los derechos humanos. Una educación para la paz y los derechos humanos estriba en la formación de valores relacionados con la forma en que las personas interactúan en sociedad y desarrollan estrategias pacificas para afrontar los conflictos sociales (Esquivel y García, 2018). Aunado a lo anterior, los modelos educativos que se construyen al interior de las universidades públicas terminan, tarde o temprano, por impactar los diseños curriculares de las universidades privadas y, por ende, aportan a toda la plataforma de la educación jurídica en un país.

Del análisis de dichos planes de estudios se encontraron los siguientes comunes denominadores:

- El promedio de asignaturas obligatorias para cumplir con todos los créditos es de 50,16, con la UABC como la que menos asignaturas requiere, con 38 , y la UACH la que más, con 59.

- El 30,9\% de las asignaturas obligatorias de los seis planes de estudios analizados se centra en las áreas de derecho privado (civil, mercantil y familiar), derecho penal y derecho social (laboral y agrario). La que más enfatiza en estas áreas es la UAT con 21, y la que menos la UNISON con 12.

- El 25,9\% de las asignaturas obligatorias corresponde a aspectos teóricos y doctrinales del derecho. La que más abarca asignaturas teóricas es la UACH con 18, y la que menos la UNISON, con 9.

- $\mathrm{El} 9 \%$ de las asignaturas obligatorias aborda las áreas de derecho constitucional y derechos humanos.

- $\quad$ El 7,3\% de las asignaturas obligatorias comprende las líneas de derecho fiscal y administrativo.

- $\mathrm{El}$ 4,7\% de las asignaturas obligatorias aborda las áreas de derecho internacional.

- El 9\% de las asignaturas obligatorias contempla materias técnicas o de fortalecimiento de herramientas personales (desarrollo de aprendizaje, técnicas de investigación, práctica jurídica, matemáticas, estadística descriptiva, redacción jurídica, etcétera). 
- Del total de planes de estudios observados, solo se desprenden 22 asignaturas novedosas o distintas al común denominador, es decir, solo el 7,3\%. Aquí se registraron áreas como derecho bancario, derechos del consumidor, derecho energético, inglés e inglés jurídico, emprendimiento y liderazgo, entre otras. El plan de estudios con más asignaturas de esta índole es el de la UANL, con 9.

- Ninguno de los planes de estudios examinados incluye alguna asignatura específica sobre derecho migratorio, a pesar de encontrarse en la frontera norte de México, ya que si bien es cierto que todos los planes incluyen el área de derecho internacional privado, en esta asignatura el tópico migratorio comprende solo uno de sus varios elementos, por lo que no es una materia especializada en ese rubro como tal.

Como se puede apreciar, los planes de estudios de la licenciatura en Derecho de las instituciones de educación superior públicas de la región norte de México tienen una notoria inclinación por las áreas tradicionales del derecho (civil, penal y laboral) y los fundamentos teóricos de la ciencia jurídica. Las ramas del derecho como lo fiscal, administrativo, constitucional, derechos humanos y derecho internacional quedan relegadas a un segundo plano, mientras que asignaturas contemporáneas que tienen una génesis más reciente como tópico jurídico tampoco tienen mucha presencia en los referidos planes de estudios.

Lo anterior limita de manera indirecta las posibilidades de inserción de los egresados de estas universidades a los mercados de trabajo, pues su instrucción y herramientas para el ejercicio profesional están constreñidas a estas áreas, en las que además los otros egresados de las instituciones de educación superior de la región también fueron capacitados. En otras palabras, se reducen las oportunidades de empleo y las que existen se ofrecen para un gran conglomerado de recién egresados que están instruidos para operar con mayor énfasis en las áreas tradicionales del derecho.

\section{Derecho y migración: Un binomio necesario para los estudios superiores en la región norte de México y para el ejercicio profesional del derecho}

La movilidad humana es un fenómeno cada vez más constante en las realidades contemporáneas del mundo y México no ha sido la excepción, pues en la actualidad constituye no solo un lugar de origen, tránsito y retorno de personas migrantes, sino también de refugio temporal y definitivo. El derecho, desde su ámbito de análisis, ha comenzado a interesarse por estudiar estas prácticas humanas y ha considerado con mayor regularidad a la migración como uno de sus tópicos a investigar (Gutiérrez López, 2019), principalmente en las regiones fronterizas.

Existen varias perspectivas jurídicas desde las que puede abordarse el fenómeno migratorio. En primer término, desde el derecho internacional - tanto público como 
privado- para comprender las relaciones entre los Estados y sus acuerdos para la gestión de diferentes temas, incluidos el migratorio, así como para comprender los procesos administrativos -internos como internacionales- de regularización migratoria, naturalización, refugio, etcétera. En segundo término, desde los derechos humanos, para analizar los diferentes instrumentos jurídicos internacionales que protegen a las personas migrantes, las vías de protección internacional y las acciones tendientes a ejecutar estos mecanismos. Una mezcla de ambas perspectivas podría servir para diseñar una carta descriptiva de una asignatura en Derecho Migratorio, necesaria para las licenciaturas en Derecho de las universidades del norte de México.

La franja norte de México se caracteriza por sus dinámicas transfronterizas, que incluyen el ingreso y salida de miles de personas diariamente, la presencia de empresas binacionales y maquiladoras extranjeras, además de ser un espacio recurrente para personas en contexto de movilidad, tanto de mexicanos como personas de otros países que tienen como finalidad acceder a territorio estadounidense. Este contexto exige, entre otras cosas, la necesidad de contar con personas que tengan conocimientos jurídicos que den respuesta desde diferentes frentes a estas realidades sociales, por ejemplo, en la aduana mexicana, en las agencias aduanales privadas, en la orientación laboral y fiscal en torno a empresas binacionales y extranjeras, en la regularización migratoria de quienes vienen a trabajar a esta región y, por supuesto, en las organizaciones civiles protectoras de personas migrantes, que velan por sus derechos humanos en una zona de mucha afluencia.

Para autores como Canales (2016), el tópico de la migración y principalmente la migración internacional ya no es más un tema exclusivo de los académicos, ya que se ha ido adentrando a las discusiones políticas en el plano global, de manera que constituyen, junto al calentamiento global y a las desigualdades regionales, los temas de mayor coyuntura a nivel mundial y, por ende, no deben de quedar aislados del interés universitario, ya que las instituciones educativas deben ajustarse a los procesos de cambio derivados de la globalización (Cornejo, 2012). El hecho de que el fenómeno migratorio haya transitado de las reflexiones académicas a las políticas abre camino al terreno jurídico y se vuelve un nicho de oportunidad para trabajar el tema desde propuestas legislativas, asesoría técnica y litigio estratégico en esta disciplina.

El estudio del derecho migratorio en las instituciones de educación superior del norte de México abriría el espacio para que los egresados pudieran integrarse a áreas laborales, como las autoridades migratorias mexicanas en sus diferentes departamentos operativos, en oficinas legales privadas especializadas en el rubro migratorio - que no proliferan en estas regiones del país - e incluso en las organizaciones de la sociedad civil y organismos internacionales protectores de derechos humanos de personas migrantes. En otras palabras, comprendería los tres grandes campos de inserción laboral de los egresados de las licenciaturas en Derecho (público, privado y social). 
La integración del derecho migratorio a los planes curriculares de los programas de licenciatura de las universidades públicas no solo aportaría a los requerimientos del mercado de trabajo de esta región del país en términos de temáticas migratorias y transnacionales, sino que también sumaría al compromiso social que tienen dichas instituciones educativas de acuerdo con sus nuevos modelos de enseñanza y aprendizaje. Como ha quedado claro en párrafos anteriores, los programas de estudio según algunos enfoques de la teoría curricular deben ser sensibles a los fenómenos y realidades sociales que rodean el entorno de las universidades.

Aunque pareciere que ya se ha desarrollado de forma robusta el debate de la migración desde la óptica del derecho con la adopción de diferentes tratados internacionales que versan sobre estos tópicos, la realidad es diametralmente opuesta. Por ejemplo, si bien México ha suscrito y ratificado la mayor parte de estos instrumentos jurídicos internacionales, tanto en materia de prerrogativas de personas migrantes y de refugiados, no se ha caminado en paralelo la adaptación de los marcos jurídicos internos a los internacionales o en la racionalidad de las dependencias y autoridades migratorias y de otra índole (Pérez, 2010). Esto deja libre el camino para el desarrollo, discusión y propuestas de nuevos juristas interesados por esta área disciplinaria.

Por lo anterior, resulta sorprendente que pese a ser la migración un asunto toral y vigente en la agenda internacional tanto en los ámbitos académico, político y jurídico, aunado a la situación contextual de las regiones fronterizas del norte de México en cuanto a sus dinámicas migratorias, las instituciones de educación superior de la frontera norte no hayan adecuado sus planes de estudio en función de estos nuevos requerimientos temáticos. De la revisión de los planes de estudios de las seis universidades mexicanas analizadas, en ninguna se presenta una asignatura que aborde el fenómeno migratorio desde el punto de vista legal.

Es evidente que la estrategia no solo debe limitarse a la inclusión del derecho migratorio a los planes curriculares, pues necesita ir acompañada de la conformación de redes con instituciones públicas, sector industrial, organismos internacionales y organizaciones de la sociedad interesados en perfiles juristas especializados en el área migratoria. No solo con la finalidad de abrir los espacios para la futura integración laboral de los egresados, sino también para la comprensión del contexto social, el trabajo colaborativo y la creación de estudios colectivos que abonen a la situación de la región en este rubro, sobre todo si tomamos en consideración que en la actualidad la vinculación universitaria es «la mejor expresión de una integración creativa universidad-sociedad» (Cedeño y Machado, 2012: 372).

Estas deficiencias curriculares no solo afectan la inserción al mercado laboral de los egresados, ya que no existe lo que Álvarez, González y López (2009) llaman «adecuación entre oferta y demanda educativa», sino al posicionamiento de estas instituciones como referentes consultivos y de opinión ante los sectores público y privado $y$, por ende, a la acreditación de sus programas como de calidad por los organismos 
evaluadores correspondientes. Ante la ausencia de licenciados en Derecho expertos en asuntos migratorios, se recurre a profesionistas de otras áreas (sociólogos, antropólogos, internacionalistas, etcétera) para que cubran dichos espacios, sin que necesariamente sean expertos en la interpretación, aplicación y discusión de los aspectos normativos y jurídicos.

\section{Espacios laborales y experiencias profesionales: Dos casos en Tijuana, Baja California}

A efecto de evidenciar la pertinencia de incluir el binomio derecho y migración en las licenciaturas en Derecho de las universidades públicas de la región norte de México, se llevaron a cabo dos entrevistas semiestructuradas a profesionistas egresados de algunas de estas instituciones de educación superior de sus programas de Derecho, quienes en la actualidad se dedican a diferentes áreas asociadas a esta línea profesional. El primer entrevistado es un consultor jurídico en materia migratoria y derecho deportivo, con una experiencia de diez años, encargado de tramitar procedimientos de los futbolistas extranjeros que llegan a jugar al equipo profesional de la ciudad de Tijuana de la Liga Mexicana de Futbol. La segunda entrevistada labora en una oficina gubernamental que tramita solicitudes de personas refugiadas en la ciudad de Tijuana, y tiene alrededor de dos años integrada al campo del derecho migratorio.

Ambas personas entrevistadas se integraron a esta área profesional por iniciativa propia, ya que tenían interés en temas como las relaciones exteriores, el primer entrevistado; y derechos humanos, la segunda. Sin embargo, ambos estiman que el plan curricular de la licenciatura en Derecho de la Universidad Autónoma de Baja California - que es donde estudiaron- es escueto respecto de los requerimientos pragmáticos del derecho migratorio, pues aunque tuvieron algunos cursos que de forma indirecta abonaron a su preparación antes de laborar en esta área, éstos parten de premisas muy generales, teóricas y no encaminadas al entendimiento de las múltiples dimensiones del fenómeno migratorio y su práctica legal. Por ello, consideran que una opción viable es independizar el derecho migratorio y extraerlo del derecho internacional privado, de los derechos humanos y del derecho administrativo, para que se retome no solo desde un punto de vista secundario, sino uno más integral.

Las dos personas entrevistadas reconocen un leve crecimiento en el número de abogados que actualmente se dedican al derecho migratorio, aunque comparado con otros campos jurídicos sigue siendo reducido. El consultor jurídico entrevistado considera que es probable que no se rebasen en la ciudad de Tijuana los diez bufetes jurídicos especializados en estos servicios. Esta situación, aunada a la vasta demanda de servicios migratorios tanto de personas que son de Estados Unidos pero viven en Tijuana, quienes tienen o desean la doble nacionalidad, así como de personas extranjeras que llegan a esta ciudad del norte de México, hace que los costos de estos servi- 
cios se encarezcan tanto que haya quienes no puedan pagarlos, o que estos despachos generen grandes utilidades en comparación con otros bufetes especializados en áreas muy sobrepobladas, a los que les cuesta trabajo hacerse de servicios.

Sumado a la ausencia de esta línea temática en el plan curricular, de la información proporcionada por ambas personas entrevistadas queda de manifesto que tampoco se han construido redes sólidas con los actores especializados en derecho migratorio (bufetes jurídicos, organismos internacionales, organizaciones de la sociedad civil y autoridades migratorias) que pudiesen operar como mecanismos de fomento para los estudiantes de esta licenciatura, a efecto de que contemplen como una opción dedicarse laboralmente en un futuro a este campo profesional. Incluso el consultor jurídico entrevistado ve factible que, si fuese complejo rediseñar el plan de estudios, otra opción podría ser ofertarlo como programa de posgrado para quien decida especializarse una vez terminada su licenciatura.

Para ambas personas entrevistadas resulta sorprendente que siendo Tijuana la frontera más visitada del mundo, no exista el interés curricular por anexar el derecho migratorio a la carga académica, pues esto no solo ayudaría a resolver un problema de sobrepoblación ocupacional en ciertas áreas del derecho y de bajos salarios, sino que además aportaría asistencia en la comunidad a la mejor regulación y gestión de las diversas dinámicas migratorias que se presentan en esta ciudad y en las que se caracterizan por ser fronterizas. La persona entrevistada que labora en una oficina gubernamental prevé que sí hay mucho trabajo en esta línea profesional y que por supuesto que enfatizar en ella en los planes de estudio de las universidades públicas sumaría al problema de integración laboral que viven los jóvenes egresados de Derecho, pues ésta y otras áreas (derecho ambiental, energético, etcétera) son continuamente ignoradas; pero, al tratarse de Tijuana, lo coyuntural e imprescindible es sin duda el derecho migratorio.

Como se puede apreciar, el derecho migratorio conecta con muchas otras subdisciplinas: derecho deportivo, derecho internacional de los refugiados, derecho administrativo, etcétera. Estos espacios no están siendo ocupados por muchos profesionistas del derecho, a pesar de haber mucho trabajo por realizar. Estas experiencias de los espacios en que puede desempeñarse un abogado dentro del derecho migratorio y de las experiencias profesionales de las dos personas entrevistadas dan muestra de lo menester que resulta la inclusión de esta temática especializada en los planes curriculares de Derecho en México y, principalmente, de las universidades de la región norte del país.

\section{Consideraciones finales}

La inserción al mercado laboral de quienes egresan de alguna licenciatura en Derecho y obtienen su cédula profesional para ejercer en México representa en la actuali- 
dad todo un reto para el Estado mexicano y para las instituciones de educación superior. Hay una gran proliferación de nuevos licenciados en Derecho cada seis o cuatro meses y las áreas de recepción en el sector del trabajo no necesariamente brindan los espacios u oportunidades que se requieren para atender a esa población profesional.

Esta sobrepoblación de licenciados está determinada por la flexibilidad que existe desde los marcos normativos de la educación para ofrecer esta carrera profesional a nivel superior. Una flexibilidad que además incide en la baja calidad de los programas de estudio y su forma de operar, pues ante lo laxo de los requerimientos legales y educativos, la mayor parte de las universidades públicas o privadas que ofrecen la licenciatura en México lo hace bajo un programa que no se encuentra acreditado ante algún organismo evaluador de la educación superior.

Existe una gran ventaja dentro de la ciencia jurídica, que estriba en la amplitud de su campo de acción y que permite a los licenciados en Derecho tener una vasta gama de posibilidades para elegir en qué área disciplinaria o sector desempeñarse. Sin embargo, las ramas jurídicas tradicionales siguen manteniendo una hegemonía en las universidades del país, por lo que estas últimas se han distanciado de las nuevas reflexiones, de las áreas novedosas y de la visión del derecho como un mecanismo de asistencia o apoyo para la resolución de los retos globales que acompañan a la sociedad actual.

Es en esta coyuntura cuando las universidades de México deberían aprovechar para abastecerse de temáticas actuales y ofrecerlas como asignaturas dentro de sus planes de estudio, a efecto de diversificar las áreas en que los egresados pueden laborar y romper al mismo tiempo con la hegemonía de las tradicionales, pues esto al final complejiza la inserción de estas personas al mercado de trabajo, ya que la mayoría pretende dedicarse a las mismas actividades y disciplinas, al tiempo que, bajo esta estrategia curricular, las instituciones de educación superior se trasformarían en un referente consultivo o de opinión en materia jurídica y en temas de coyuntura e impacto.

La región del norte de México es un espacio con características propias que definen su contexto y la diferencian de otras zonas del país. En ella convergen diversos fenómenos sociales, demográficos y culturales que demandan acciones específicas y análisis idóneos para la gestión de estas realidades. El tema migratorio es una de esas realidades y el derecho una de las disciplinas que abonan - o deberían abonar- al debido tratamiento de estos fenómenos.

La ausencia de asignaturas especializadas en migración en los planes de estudios y de redes de colaboración con los actores clave del fenómeno migratorio de las universidades públicas más representativas de la región norte de México muestra la poca preocupación de estas instituciones por la integración al mercado laboral de sus egresados. A pesar de ser un nicho de oportunidad fructífero por las dinámicas migratorias que experimentan estas regiones, se ha decidido relegarlas de los 
programas de estudio. Incluso los espacios de trabajo con énfasis en los estudios migratorios han comenzado a ser ocupados por profesionales de las ciencias sociales, quienes no dominan la interpretación y aplicación de las leyes e instrumentos legales.

En la medida en que las instituciones de educación superior de la frontera norte de México se ocupen de incluir la línea temática de derecho migratorio - entre algunas otras- en sus mapas curriculares de la licenciatura en Derecho, se extenderá el campo de inserción laboral de los profesionales de esta área. De lo contrario, las vicisitudes que ya existen para que se integren laboralmente los egresados de las ciencias jurídicas, como producto de la sobrepoblación, por un lado, y de la hegemonía de las ramas tradicionales por el otro, se multiplicarán y harán cada vez más adverso el panorama. Es por ello que en este trabajo se estima que el binomio derecho y migración es una respuesta curricular pronta, práctica y posible a la complejidad de la sobrepoblación de licenciados en Derecho en México y en concreto en el norte del país, así como al problema de su integración al mercado de trabajo bajo condiciones laborales dignas.

\section{Referencias}

Álvarez, Pedro, Alfonso González y David López (2009). «La enseñanza universitaria y la formación para el trabajo: Un análisis desde la opinión de los estudiantes». Paradígma, 30 (2): 7-20. Disponible en https://bit.ly/313jN98.

Barraza, Jesús (2016). «El trasfondo del enfoque por competencias en México». En Jesús Trujillo y José García (coordinadores), Desarrollo profesional docente: Reforma educativa, contenidos curriculares y procesos de evaluación (pp. 33-42). Chihuahua: Escuela Normal Superior Profr. José E. Medrano R.

Camarena, Beatriz y Delisahé Velarde (2009). «Educación superior y mercado laboral: Vinculación y pertinencia social. ¿Por qué? y ¿para qué?». Estudios sociales, 17: 105-125. Disponible en https://bit.ly/2YOfhII.

Canales, Alejandro (2016). Debates contemporáneos sobre migración internacional: Una mirada desde América Latina. Ciudad México: Miguel Ángel Porrúa.

Cedeño, Julio y Evelio Machado (2012). «Papel de la extensión universitaria en la transformación local y el desarrollo social». Humanidades Médicas, 12 (3): 37139o. Disponible en https://bit.ly/3hJ6KiS.

CEEAD, Centro de Estudios sobre la Enseñanza y el Aprendizaje de Derecho (2018). Informe anual 2018: Transformando la enseñanza del derecho en México. Monterrey. Collazo, Carmen (2014). "La responsabilidad social universitaria (RSU) un compromiso ineludible». Kálathos, Revista Transdisciplinaria Metro-Inter, 7 (2): 1-13. Disponible en https://bit.ly/313x2Xk. 
Contreras, Óscar y Alfredo Hualde (2015). La situación actual de las ciencias sociales en el noroeste de México. Tijuana: Consejo Mexicano de Ciencias Sociales y Foro Consultivo Científico y Tecnológico.

Cornejo, Juan (2012). «Retos impuestos por la globalización a los sistemas educativos latinoamericanos». Revista Mexicana de Investigación Educativa, 17 (52): 15-37. Disponible en https://bit.ly/3dogv5M.

DíAz, Frida (1993). «Aproximaciones metodológicas al diseño curricular hacia una propuesta integral». Tecnología y Comunicación Educativas, 21: 19-39. Disponible en https://bit.ly/37Nblff.

EsQuivel, Cristela y Mirna García (2018). «La educación para la paz y los derechos humanos en la creación de valores para la solución de conflictos escolares». Justicia, 23 (33): 256-270. DOI: 10.17081/just.23.33.2892.

GHAI, Dharam (2003). «Trabajo decente: Concepto e indicadores». Revista Internacional del Trabajo, 122 (2): 125-16o. Disponible en https://bit.ly/3enXT3W.

GonzÁlez, Manuela, María Gabriela Marano, Carola Bianco y María Cecilia Carrera (2011). «Estado del arte de la educación jurídica: A diez años del Congreso Nacional de Sociología Jurídica». Academia: Revista sobre Enseñanza del Derecho, 9 (17): 175-218. Disponible en https://bit.ly/2YLLkIJ.

GonzÁlez, Sebastián y Elisa Muñoz (2010). «Análisis de las competencias de los estudiantes de Derecho en los nuevos planes de estudio de Andalucía en el EEES XXI». Revista de Educación, 12: 147-162. Disponible en https://bit.ly/3dlUAsG.

GutiéRREZ LóPEZ, Eduardo Elías (2019). «Análisis sociojurídico de la percepción de migrantes centroamericanos sobre derechos humanos en la frontera sur mexicana». Tesis para postular al grado de doctor en Estudios de Migración. El Colegio de la Frontera Norte, Tijuana. Disponible en https://bit.ly/2YhyRoX.

Medina, Emma y Érika Valdez (2011). «El proceso de acreditación en programas de educación superior: Un estudio de caso». Omnia, 17 (2): 53-70. Disponible en https://bit.ly/2NgrTTA.

Neave, Guy (1998). Debate temático: Autonomía, responsabilidad social y libertad académica. París: Unesco.

PÉrez, Nancy (2010). «La importancia de una ley migratoria en México». Migración $y$ Desarrollo, 18 (15): 187-202. Disponible en https://bit.ly/37NQVmD.

VAllaeys, François (2014). «La responsabilidad social universitaria: Un nuevo modelo universitario contra la mercantilización». Revista Iberoamericana de Educación Superior, 5 (12): 105-117. Disponible en https://bit.ly/2YhzQ19.

\section{Sobre el autor}

EduARdo ElÍAs GutiÉRrez López es maestro en Ciencias Jurídicas por la Universidad Autónoma de Baja California, México, y doctor en Estudios de Migración por El 
Colegio de la Frontera Norte, México. Actualmente es profesor de tiempo completo en la Facultad de Derecho Tijuana de la Universidad Autónoma de Baja California y profesor de Posgrado en la Universidad de las Californias Internacional y en el Centro de Enseñanza Técnica y Superior de Tijuana. Su correo electrónico es elias. gutierrez@uabc.edu.mx. (D) https://orcid.org/oooo-0oo2-9171-8813. 
La Revista Pedagogía Universitaria y Didáctica del Derecho (RPUDD) es una publicación científica semestral que contribuye a la reflexión multidisciplinaria sobre pedagogía universitaria y didáctica del derecho, para la formación y consolidación de esta área de investigación; así como a la difusión de prácticas innovadoras en la enseñanza-aprendizaje del derecho considerando el contexto nacional e internacional. Es una publicación electrónica internacional con una codirección entre Brasil y Chile.

\author{
DIRECTORA \\ María Francisca Elgueta Rosas \\ Universidad de Chile \\ DIRECTOR \\ Renato Duro Dias \\ Universidad Federal de Rio Grande, Brasil \\ SITIO WEB \\ pedagogiaderecho.uchile.cl \\ CORREO ELECTRÓNICO \\ rpedagogia@derecho.uchile.cl \\ LICENCIA DE ESTE ARTÍ́CULO
}

Creative Commons Atribución Compartir Igual 4.0 Internacional

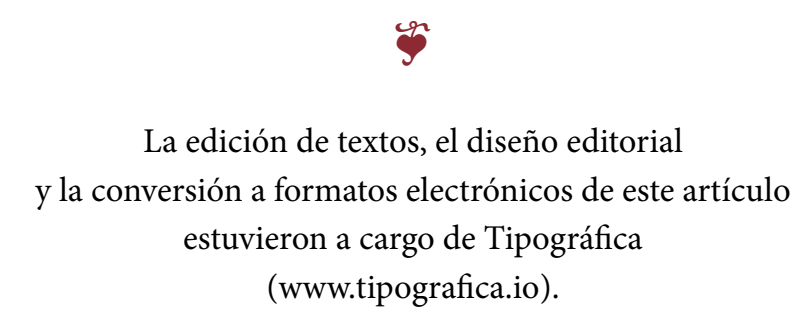

